\section{Nouvelles de la SSDV Neues aus der SGDV}

\section{Société Suisse de Dermatologie et Vénéréologie Organigramme 2005 (état janvier 2005)}

\section{Schweizerische Gesellschaft für Dermatologie und Venerologie}

Organigramm 2005 (Stand Januar 2005)

\section{Président/Präsident}

$\mathrm{Dr} \quad$ Félix Gueissaz

33, avenue du 1er Mars

2000 Neuchâtel

Tél. $0327214575 / 80$

Fax 0327214588

felix.gueissaz@net2000.ch

Président-elect/Präsident-elect

\begin{tabular}{ll}
\hline Prof. Dr Peter Itin & Kantonsspital Aarau \\
& 5000 Aarau \\
& Tél. 0628386952 \\
& Fax 0618386953 \\
& Peter.itin@ksa.ch \\
& \\
\hline Vice-président/Vize-Präsident ${ }^{1}$ & Spital Zollikerberg \\
\hline Prof. Dr Alfred Eichmann & 8125 Zollikerberg \\
& Tél. 01 397 2814 \\
& Fax 013972112 \\
& prof.eichmann@ \\
& spitalzollikerberg.ch
\end{tabular}

Trésorière/Quästorin

Dr Carmen Laetsch

\section{1, Fläscherstrasse}

7310 Bad-Ragaz

Tél. 0813022228

Fax 0813021774

Carmen.Laetsch@hin.ch

\section{Membres du Comité/Vorstandsmitglieder}

\begin{tabular}{|c|c|c|c|}
\hline Prof. & Braathen & Lasse R. & lasse.r.braathen@insel.ch \\
\hline Prof. & Burg & Günter & burg@derm.unizh.ch \\
\hline Prof. & Rufli & Theo & trufli@uhbs.ch \\
\hline Prof. & Panizzon ${ }^{1}$ & Renato & renato.panizzon@chuv.hospvd.c \\
\hline Prof. & Saurat & Jean-Hilaire & marion.bonna@hcuge.ch \\
\hline Dr & Bänninger & Rolf & rolf.baenninger@bluewin.ch \\
\hline $\mathrm{Dr}$ & Cajacob & Andreas & andreas.cajacob@hin.ch \\
\hline $\mathrm{Dr}$ & Gabbud $^{1}$ & Jean-Paul & jgabbud@bluewin.ch \\
\hline Dr & Grillet & Jean-Pierre & jgrillet@vtxnet.ch \\
\hline $\mathrm{Dr}$ & Laetsch & Carmen & Carmen.Laetsch@hin.ch \\
\hline PD Dr & Lautenschlager & Stephan & $\begin{array}{l}\text { stephan.lautenschlager@triemli. } \\
\text { stzh.ch }\end{array}$ \\
\hline $\mathrm{Dr}$ & Mainetti & Carlo & info@carlomainetti.ch \\
\hline Dr & $\begin{array}{l}\text { Mancarella- } \\
\text { Eberhardt }\end{array}$ & Anna & amancarella.eberhardt@hin.ch \\
\hline
\end{tabular}

${ }^{1}$ Membres du Bureau du Comité/Mitglieder des Ausschusses

\section{Secrétariat/Sekretariat}

\begin{tabular}{ll}
\hline Mme/Frau Pongratz Guntli Monica & 33, avenue du 1er Mars \\
& 2000 Neuchâtel \\
& Tel. 0327214260 \\
& Fax 0327214588 \\
& F: ssdv@net2000.ch \\
& D: sgdv@net2000.ch
\end{tabular}

\section{Vérificateurs des comptes/Rechnungsrevisoren}

\begin{tabular}{llll}
\hline $\mathrm{Dr}$ & Ingold & Rolf & ringold@bluewin.ch \\
$\mathrm{Dr}$ & Kägi & Martin & praxis.kaegi@hin.ch
\end{tabular}

Membres d'honneur/Ehrenmitglieder

\begin{tabular}{llll}
\hline Dr & Bigliardi & Paul & 8245 Feuerthalen \\
Prof. & Braun-Falco & Otto & 81545 München \\
Prof. & Christophers & Enno & 24098 Kiel \\
Prof. & Delacrétaz & Jean & 1003 Lausanne \\
Dr & Favre & François & 2514 Ligerz \\
Prof. & Frenk & Edgar & 1820 Montreux \\
Dr & Gilliet & François & 6500 Bellinzona \\
Prof. & Jung & Ernst G. & 68167 Mannheim \\
Prof. & Krebs & Alfred & 3012 Bern \\
Dr & Kull & Eugen & 8704 Herrliberg \\
Prof. & Laugier & Paul & 1206 Genève \\
Dr & Mevorah & Barukh & 64365 Tel-Aviv \\
Dr & Perroud & Henri M. & 1700 Fribourg \\
Prof. & Plewig & Gerd & 80337 München \\
Dr & Ramelet & Albert A. & 1003 Lausanne \\
Prof. & Schnyder & Urs W. & 8006 Zurich \\
Prof. & Schuppli & R. & 4059 Basel \\
Prof. & Schwarz & Kaspar J. & 8044 Zurich \\
Prof. & Wüthrich & Brunello & 8091 Zurich \\
& & &
\end{tabular}

Membres correspondants/Korrespondierende Mitglieder

\begin{tabular}{llll}
\hline Prof. & Borelli & Siegfried & Davos, CH \\
Prof. & Bruckner-Tuderman Leena & Freiburg, D \\
Prof. & Dubertret & Louis & Paris, F \\
Prof. & Elsner & Peter & Jena, D \\
Prof. & Grosshans & Edouard & Strasbourg, F \\
Prof. & Humbert & Philippe & Besançon, F \\
Prof. & Kaufmann & Roland & Frankfurt/Main, D \\
Prof. & Kerl & Helmut & Graz, A \\
Prof. & Mumcuoglu & Kosta Y. & Jerusalem, Israël \\
Prof. & Schöpf & Erwin & Freiburg, D \\
Prof. & Stingl & Georg & Wien, A
\end{tabular}

Délégué SSDV à la Chambre médicale/Ärztekammer-Delegierter SGDV Dr Gueissaz félix felix.gueissaz@net2000.ch

Délégués SSDV à la FMS/FMS-Delegierte SGDV

\begin{tabular}{llll}
\hline $\mathrm{Dr}$ & Gueissaz & Félix & felix.gueissaz@net2000.ch \\
$\mathrm{Dr}$ & Grillet & Jean-Pierre & jgrillet@vtxnet.ch \\
$\mathrm{Dr}$ & Schmidli & Jürg & praxis-schmidli@bluewin.ch
\end{tabular}

Conseil de Déontologie/Ehrenrat

\begin{tabular}{llll}
\hline Dr & Perroud & Henri (prés.) & henri.m.perroud@hin.ch \\
Prof. & Rufli & Theo & trufli@uhbs.ch \\
$\mathrm{Dr}$ & Knüsel & Jörg & jknuesel@hin.ch \\
$\mathrm{Dr}$ & Montaldi & Giovanna & montaldi@dermastudio.ch \\
$\mathrm{Dr}$ & Suter & Hans & \\
& &
\end{tabular}

Commission SSDV des intérêts professionnels/SGDV-Kommission zur Wahrung der Standesinteressen

\begin{tabular}{|c|c|c|}
\hline $\mathrm{Dr}$ & Grillet & Jean-Pierre (prés.) \\
\hline & & jgrillet@vtxnet.ch \\
\hline $\mathrm{Dr}$ & Adatto & info@skinpulse.ch \\
\hline $\mathrm{Dr}$ & Gütling & guetling@bluewin.ch \\
\hline $\mathrm{Dr}$ & Schmidli & praxis-schmidli@bluewin.ch \\
\hline
\end{tabular}

Commission SSDV pour la formation post-graduée et continue/ SGDV-Kommission für Weiter- und Fortbildung

\begin{tabular}{|c|c|c|c|}
\hline Prof. & Eichmann & Alfred (prés.) & $\begin{array}{l}\text { prof.eichmann@ } \\
\text { spitalzollikerberg.ch }\end{array}$ \\
\hline $\mathrm{Dr}$ & Cajacob & Andreas & andreas.cajacob@hin.ch \\
\hline $\mathrm{Dr}$ & Perroud & Henri & henri.m.perroud@hin.ch \\
\hline \multicolumn{4}{|c|}{$\begin{array}{l}\text { Commission SSDV de la promotion de qualité/SGDV-Kommission für } \\
\text { Qualitätssicherung }\end{array}$} \\
\hline \multirow{4}{*}{$\begin{array}{l}\text { Prof. } \\
\text { Dr } \\
\text { Dr }\end{array}$} & Braathen & Lasse R. (prés.) & lasse.r.braathen@insel.ch \\
\hline & Laetsch & Carmen & Carmen.Laetsch@hin.ch \\
\hline & Mancarella- & & \\
\hline & Eberhardt & Anna & amancarella.eberhardt@hin.ch \\
\hline
\end{tabular}


Commission SSDV des examens de spécialistes FMH/SGDV-Prüfungskommission für die Facharztprüfung FMH

\begin{tabular}{lll}
\hline Prof. & Büchner & Stanislaw (prés.) \\
Prof. & Hohl & Daniel \\
Prof. & Trüeb & Ralph \\
PD Dr & Borradori & Luca \\
Dr & Camenzind & Michel \\
$\mathrm{Dr}$ & Meyrat & René \\
$\mathrm{Dr}$ & Pelloni & Francesco \\
$\mathrm{Dr}$ & Perroud & Henri \\
Vacant/vakant & \\
Vacant/vakant &
\end{tabular}

sbuechner@uhbs.ch

daniel.hohl@chuv.hospdv.ch ramitru@derm.unizh.ch Luca.Borradori@hcuge.ch mcamenzind@hotmail.com rene.meyrat@hin.ch fpelloni@hin.ch henri.m.perroud@hin.ch

Commission SSDV des médias/SGDV-Medienkommission

\begin{tabular}{llll}
\hline Prof. & Eichmann & Alfred (prés.) & $\begin{array}{l}\text { prof.eichmann@ } \\
\text { spitalzollikerberg.ch }\end{array}$ \\
Dr & Grillet & Jean-Pierre & jgrillet@vtxnet.ch \\
Dr & Braun & Ralph & braun@melanoma.ch \\
Dr & Cajacob & Andreas (rédact. D) & andreas.cajacob@hin.ch \\
Dr & Pelloni & Francesco (rédact. I) & fpelloni@hin.ch \\
Dr & Tapernoux & Bernard (rédact. F) & bernard.tapernoux@hin.ch
\end{tabular}

\section{Groupes de travail SSDV/SGDV-Arbeitsgruppen}

\section{AcnélAkne}

$\begin{array}{lll}\text { Dr Harms } & \text { Monika } & \text { monika.harms@bluewin.ch } \\ \begin{array}{l}\text { Allergologie } \\ \text { Prof. Hauser }\end{array} & \text { Conrad } & \begin{array}{l}\text { Conrad.Hauser@medecine. } \\ \text { unige.ch }\end{array} \\ \begin{array}{l}\text { Andrologie } \\ \text { Dr Sigg }\end{array} & \text { Christian } & \text { dr.sigg@bluewin.ch } \\ \text { Dermatochirurgie } & \text { Erich } & \text { dhb@medcom.ch } \\ \text { Dr Küng } & \end{array}$

Dr Küng Erich dhb@medcom.ch

Dermatohistopathologie

Prof. Büchner Stanislaw stanislaw.buechner@hin.ch

Dermatopédiatrie/Pädiatrische Dermatologie

Prof. Hohl Daniel daniel.hohl@chuv.hospdv.ch

Les infirmières en dermatologie et les assistantes médicales/Dermatologiekrankenschwestern und Medizinassistentinnen

Vacant/vakant

SCDRG Swiss Contact Dermatitis Research Group

$\begin{array}{llll}\text { Dr } & \text { Simon } & \text { Dagmar } & \text { dagmar.simon@insel.ch } \\ \text { Dr } & \text { Sigg } & \text { Rita } & \text { praxissiggrit@bluewin.ch }\end{array}$

Trichologie

Prof. Trüeb Ralph ralph.trueeb@usz.ch

Présidents des Groupements régionaux de dermatologues Präsidenten der regionalen Dermatologenverbände

AG Aargauer Dermatologengesellschaft (1985)

DrHofer Thomas thomas.hofer@active.ch

Verein Kantonaler Bernischer Dermatologen (VKBD; 1978)

DrSigrist Urs urs.sigrist@hin.ch

BL Fachgruppe Dermatologie und Venerologie des

Kantons Baselland (2004)

Dr Pletscher Martin martin.pletscher@hin.ch Dermatologen/innen des Kantons Basel-Stadt

Dr Flückiger Ruedirhedi.flueckiger@hin.ch

FR Groupement Fribourgeois des Dermatologues (GFD; 1994)

Dr Schibli Hugo hugo.schibli@hin.ch

GE Groupe des Dermatologues Genevois (GDG; 1989)

Dr Krischer Joachim joachim.krischer@latour.ch

NE Groupement des Dermatologues de Neuchâtel et environs (GDNE; 1991)

DrFaivre Ghislain ghislain-faivre@hispeed.ch

SO Dermatologen des Kantons Solothurn (1990)

Dr Bloch Peter H. PeterH.Bloch@hin.ch

TG Gruppe Freipraktizierende Dermatologen des Kantons Thurgau (2004)

Drvan Roojen Menno_menno.vanroojen@hin.ch

Societa Ticinese di Dermato-Venereologia (STDV; 1987)

Dr Bianchi Enrica ebianchi@hin.ch

VD Groupement des Dermatologues praticiens de la Société Vaudoise de Médecine (GDV; 1991)

DrZurn André andre.zurn@vtxnet.ch
VS Groupement des Dermatologues Valaisans (1989)

DrGianadda Bernard Bernard.Gianadda@hin.ch

Dr Suard André

Bernard.Gianadda

Zürcher Dermatologen-Gesellschaft (ZDG; 1958)

Dr Gütling Michael guetling@bluewin.ch

Conseil de la fondation/Stiftungsrat «Schweizerische Stiftung zur Bekämpfung der Geschlechtskrankheiten"

PD Dr Lautenschlager Stephan stephan.lautenschlager@triemli.

Dr Mainetti stzh.ch

Commission ad hoc Dermatohistopathologie/

Dermatohistopathologie-Kommission

\begin{tabular}{llll}
\hline Prof. & Büchner & Stanislaw (prés.) & sbuechner@uhbs.ch \\
Prof. & Burg & Günter & burg@derm.unizh.ch \\
Prof. & Panizzon & Renato & renato.panizzon@chuv.hospvd.ch
\end{tabular}

Dr_De Viragh Pierre pierredeviragh@bluewin.ch

Membres de la Commission de prévention des cancers cutanés/Dermatologen, Mitglieder der Kommission Früherkennung Hautkrebs

Prof. Panizzon Renato (prés.) renato.panizzon@chuv.hospvd.ch

Prof. Burg Günter burg@derm.unizh.ch

Prof. Dummer Reinhard reinhard.dummer@usz.ch

PD Dr Brand Christophe U. christoph.brand@ksl.ch

Dr Braun Ralph braun@melanoma.ch

Dr Gabbud Jean-Paul jgabbud@bluewin.ch

Vacant/vakant

Délégués SSDV aux commissions de la FMH/Delegierte der SGDV in den Kommissionen der FMH

Commission de la formation post-graduée et continue/Kommission für Weiter- und Fortbildung

\begin{tabular}{llll}
\hline Prof. & Eichmann & Alfred & $\begin{array}{l}\text { prof.eichmann@ } \\
\text { spitalzollikerberg.ch }\end{array}$ \\
Dr & Gabbud & $\begin{array}{l}\text { Jean-Paul } \\
\text { (Mitglied des Ausschusses der KWFB) }\end{array}$
\end{tabular}

Commission des Titres/Titelkommission

Dr Mancarella- Anna amancarella.eberhardt@hin.ch
Eberhardt

Commission des établissements de la formation post-graduéel Weiterbildungsstättenkommission

\begin{tabular}{lll}
\hline Prof. Eichmann $\quad$ Alfred & $\begin{array}{l}\text { prof.eichmann@ } \\
\text { spitalzollikerberg.ch }\end{array}$
\end{tabular}

Délégués SSDV à la Commission Laser FMS/Delegierte der SGDV in der FMS-Laser-Kommission

\begin{tabular}{llll}
\hline $\operatorname{Dr}$ & Adatto & Maurice (Task Force Tarmed) \\
& & & info@skinpulse.ch \\
$\operatorname{Dr}$ & Bloch & Peter H. & PeterH.Bloch@hin.ch \\
PD Dr & Salomon & Denis & denis.salomon@hcuge.ch \\
Dr & Thürlimann & Wolfgang & wolfgang.thuerlimann@hin.ch
\end{tabular}

Commission fédérale des facultés de médecine de la Suisse/Abteilung für Ausbildungs- und Examensforschung

Prof. Braathen Lasse R. (prés.) lasse.r.braathen@insel.ch

Dr Hohl Daniel (vice-prés.) daniel.hohl@hospvd.ch

PD Dr. Borradori Luca luca.borradori@hcuge.ch

Prof. Trüeb Ralph Ralph.Trueeb@usz.ch

Dr Häusermann Peter

Délégués à I'UEMS/UEMS-Delegierte

Prof. Braathen Lasse R. lasse.r.braathen@insel.ch

Dr Gabbud Jean-Paul jgabbud@bluewin.ch

Délégué à I'EADV/EADV-Delegierter

PD Dr Borradori Luca luca.borradori@hcuge.ch

Membre de la commission pour la formation continue de I'EADV/ Mitglied der EADV-Fortbildungskommission

Dr Gabbud Jean-Paul jgabbud@bluewin.ch 
Aus Anlass des Fortbildungstages der dermatologischen Kliniken von Genf und Lausanne am 24. April 2003 in Nyon - genau 90 Jahre nach Gründung der SGDV - entstand das Projekt einer zusammenfassenden Darstellung der Geschichte der Schweizer Dermatologie, ihrer Ausbildungs- und Forschungszentren und auch der Geschichte der Gesellschaft. Im Jahr 2004 wurde dieses Projekt nun mit dem im Folgenden vorgestellten Buch zum Abschluss gebracht.

Die Dermatologie und Venerologie etablierte sich in Europa im Verlauf des 19. Jahrhunderts als Spezialfach der Medizin. Die ersten anerkannten Zentren waren London, Paris und Wien. In der Schweiz entstanden die ersten Spezialkliniken, meist im Rahmen des Ausbaus der Medizinischen Fakultäten, in den letzten zwei Jahrzehnten des 19. Jahrhunderts. Deren damalige Aufgabe war die Bekämpfung der Geschlechtskrankheiten und besonders der Syphilis, einer der wichtigsten Landplagen jener Zeit. Das vorliegende Buch beschreibt die Geschichte dieser und der seither eröffneten Kliniken, deren graduelle Umwandlung in Zentren zur Behandlung und Erforschung der immer häufiger werdenden Hautkrankheiten und die Geschichte der 1913 gegründeten Schweizerischen Gesellschaft für Dermatologie und Venerologie, die Kliniken, Polikliniken und frei praktizierende Dermatologen vereint.

A l'occasion de la Journée de formation continue des cliniques de dermatologie de Genève et de Lausanne à Nyon du 24 avril 2003 juste 90 années après la fondation de la SSDV - le projet d'un aperçu de l'histoire de la dermatologie suisse, de ses centres de formation et de recherche ainsi que de l'histoire de la Société a été conçu. En 2004, ce projet a été réalisé par le livre présenté ci-après.

La dermatologie et vénéréologie, en tant que spécialité médicale, s'est progressivement établie en Europe au cours du $19 \mathrm{e}$ siècle; les premiers centres reconnus ont été Londres, Paris et Vienne. En Suisse les premières cliniques spécialisées, rattachées aux Facultés de médecine, ont été crées vers la fin du $19 \mathrm{e}$ siècle. Leur mission principale était la lutte contre un fléau de l'époque, les maladies vénériennes et en particulier la syphilis. Ce livre retrace l'histoire de ces établissements, et de

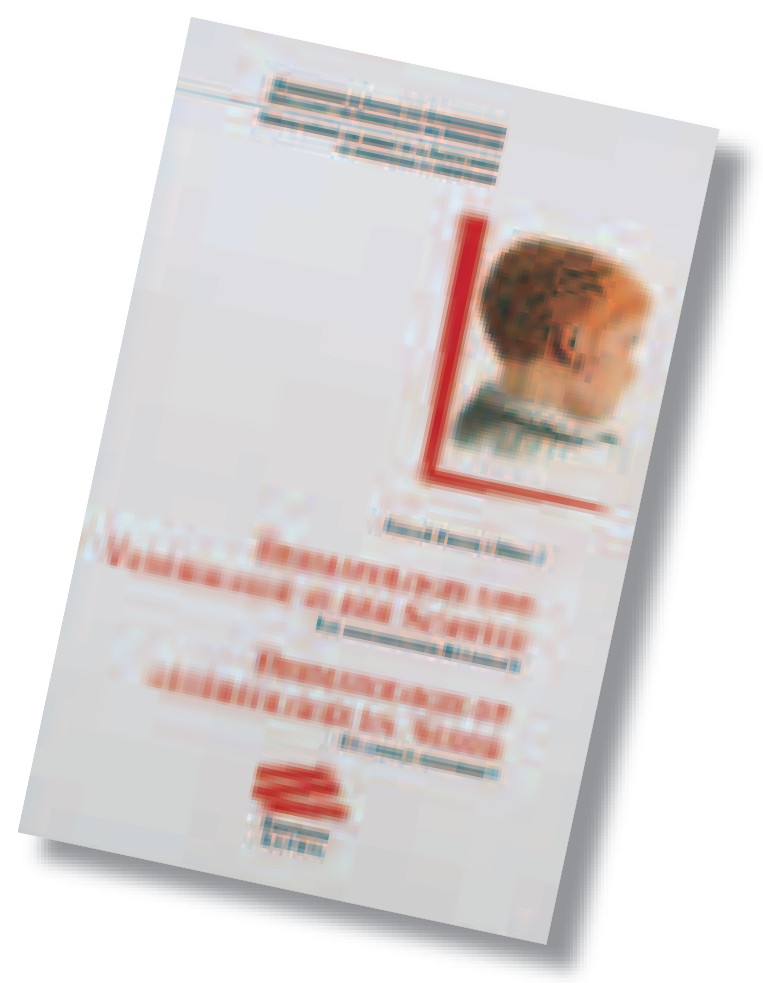

ceux ouverts depuis lors, leur transformation en centres de soins et de recherche orientés de plus en plus vers les problèmes liés au nombre croissant de maladies de la peau, ainsi que l'histoire de la Société suisse de dermatologie et vénéréologie, fondée en 1913, qui réunit les cliniques, les policliniques et les médecins spécialistes praticiens.

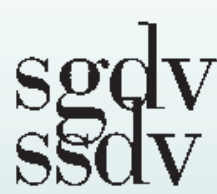

Herausgegeben im Auftrag der Schweizerischen Gesellschaft für Dermatologie und Venerologie. Ouvrage préparé sur mandat de la Société suisse de dermatologie et vénéréologie.

ISBN 2-940235-08-2

\begin{tabular}{|c|c|c|c|}
\hline Heft & Erscheinungstermine 2005 & Manuskripteingang ${ }^{1}$ & Hauptthema \\
\hline Numéro & Dates de publication 2005 & Date butoire des manuscrits ${ }^{1}$ & Sujet principal \\
\hline 1 & 14. Januar / 14 janvier & - & Geschichte der SGDV / Histoire de la SSDV \\
\hline 2 & 25. Februar / 25 février & - & \\
\hline 3 & 1. April / 1er avril & 4. Februar / 4 février & Spezialnummer SGDV / Numéro spécial SSDV \\
\hline 4 & 13. Mai / 13 mai & 18. März / 18 mars & Spezialnummer SGDV / Numéro spécial SSDV \\
\hline 5 & 24. Juni / 24 juin & 22. April / 22 avril & \\
\hline 6 & 5. August / 5 août & 27. Mai / 27 mai & Kongress SGDV / Congrès SSDV \\
\hline 7 & 16. September / 16 septembre & 15. Juli / 15 juillet & \\
\hline 8 & 28. Oktober / 28 octobre & 26. August / 26 août & \\
\hline
\end{tabular}

${ }^{1}$ Manuskripte (wenn möglich auf Deutsch und Französisch) bitte an das Redaktionssekretariat auf Diskette oder per E-Mail (als WordDokument, nicht pdf-Format): Dermatologica.Helvetica@hcuge.ch.

1 Veuillez envoyer les manuscrits (si possible en français et allemand) sur disquette ou par courrier électronique au secrétariat de rédaction (comme document Word, non pas en format pdf): Dermatologica.Helvetica@hcuge.ch.
Bitte senden Sie Ihren Text auf Deutsch und Französisch ein und fügen Sie eine Titelseite mit Ihrer vollständigen Adresse (einschliesslich Postleitzahl, Telefon- und Fax-Nummer sowie E-Mail-Adresse) bei.

Veuillez envoyer votre texte en langues française et allemande et joindre au manuscrit une page portant l'adresse complète (avec code postal, numéros de téléphone et de fax et l'adresse E-mail) de l'auteur. 


\section{Neue Mitglieder der SGDV (Oktober 2004) Nouveaux membres de la SSDV (octobre 2004)}

\section{Ordentliche Mitglieder/Membres ordinaires}

\section{Dr Stéphanie Liardet}

Spécialiste FMH en dermatologie et vénéréologie

Née le 12 avril 1972

à Estavayer-le-Lac, mariée

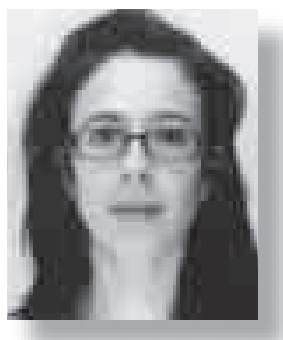

1991-1996 Etudes de médecine à la Faculté de médecine de Lausanne

1996 Diplôme de médecin, Lausanne

1997 Service de dermatologie, Prof. R. Panizzon, CHUV, Lausanne

2002 Thèse «Protection against pyrimidine dimers, p53 and 8-hydroxy-2'-deoxyguanosine expression in ultraviolet-irradiated human skin by sunscreens: Difference between UVB + UVA and UVB alone sunscreens», sous Dr Lee Ann Laurent-Applegate, Université de Lausanne

2003 Titre de spécialiste FMH en dermatologie et vénéréologie

2004 En mars, ouverture d'un cabinet médical à Lausanne

Parrainage: Dr André Zurn, Lausanne

Dr Romain Anex, Lausanne

\section{Dr. med. Erika Schuller}

Fachärztin für Dermatologie und Venerologie

Geboren am 17. Januar 1972 in Neumarkt, Rumänien, ledig

Aus- und Weiterbildung:

1991-1997 Studium der Humanmedizin an der Universität Ulm, Deutschland

1997 Dissertation «Arthroskopische Labrumrefixation Vergleich der offenen und arthroskopischen Operationstechnik bei 57 Patienten», unter Prof. Dr. Hartmuth Kiefer, Universität Ulm, Deutschland

1999 Approbation als Ärztin

2003 Facharzttitel für Dermatologie und Venerologie, Deutschland

2004 Seit Januar Praxisassistentin in der Hautarztpraxis Dr. Gütling, Winterthur

Patenschaft: Dr. Michael Gütling, Winterthur Dr. Jörg Knüsel, Winterthur

\section{Ausserordentliches Mitglied/Membre extraordinaire}

\section{Dr Constance Pournaras}

Médecin en dermatologie

et vénéréologie

Née le 17 mai 1974

à Genève, mariée

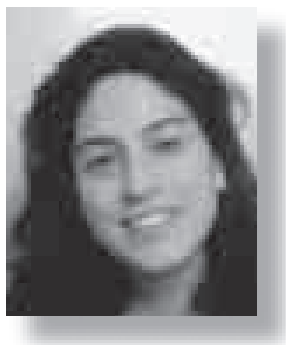

1993-1999 Etude de médecine, Faculté de médecine, Genève 1999 Diplôme de médecin, Genève

1999-2000 Thèse «Evolution de la colonisation par Staphylococcus aureus des lésions de dermatite atopique traitées par tacrolimus (FK506) topique», sous Prof. J.-H. Saurat, Genève

2002 Doctorat de médecine, Genève

2002-2004 Service de dermatologie du Prof. L. Dubertret, puis du Prof. P. Morel, Hôpital Saint-Louis, Paris

2004 Depuis mai, Service de dermatologie pédiatrique du Prof. Y. de Prost, Necker, Paris, et Service de dermatologie du Prof. J. Revuz, Créteil (gardes)

Parrainage: Prof. Lars French, Genève Dr Denis Salomon, Genève

\section{Ehrung}

Frau Dr. Barbra Fischer, Dermatologische Klinik, Universitätsspital Zürich, wurde an der Jahresversammlung der Europäischen Akademie für Dermatologie und Venerologie in Florenz für ihr Poster «Component resolved diagnosis with recombinant Malassezia allergens in atopic dermatitis» mit dem mit EUR 1000.- dotierten UriagePreis ausgezeichnet. 